\title{
Geriatrie: rationierte Medizin bei Hochbetagten?
}

\author{
M. Conzelmann
}

\footnotetext{
1 Zweifel P, Telser H. Rationierung: der Königsweg im Gesundheitswesen? Praxis 2000;89:1181-4.
}

Korrespondenz:

Dr. med. Martin Conzelmann

Felix Platter-Spital

Geriatrisches Kompetenzzentrum

Burgfelderstrasse 101

CH-4012 Basel
Eine 85jährige Patientin wird ins Spital eingewiesen wegen Exazerbation einer chronischen Lumbo-ischialgie und zunehmender Gehunfähigkeit. Bis anhin lebte die verwitwete Patientin allein und kam auch ohne fremde Hilfe in ihren Alltagsaktivitäten zurecht.

Die Abklärungen ergeben das Vorliegen einer Spinalkanalstenose und da eine zunehmende Parese an den unteren Extremitäten auftritt, wird der Patientin die operative Dekompression des Spinalkanals empfohlen. Sie willigt in den Eingriff ein, postoperativ ist die Mobilisation verzögert, da als Komplikation eine Pneumonie und ein mehrere Tage dauerndes Delir auftreten. Erst 8 Wochen nach dem Eingriff gelingt es, die Patientin wieder zu mobilisieren, und 12 Wochen nach dem Eingriff kann die Patientin wieder nach Hause zurückkehren. Da die Kostengutsprache durch die Krankenkasse trotz verschiedenster Interventionen nicht verlängert werden kann, erfolgt die Rückkehr nach Hause verfrüht. Mobil an zwei Gehstöcken, mit erheblichem Sturzrisiko verlässt sie das Spital, stürzt zu Hause auch, jedoch ohne sich zu verletzen. In der Diskussion mit der Krankenkasse werden von seiten der Kasse zur Begründung keiner weiteren Kostengutsprache Argumente wie «man operiert doch wohl kaum jemanden mit 85 Jahren am Rücken» ins Feld geführt.

Rationierung von medizinischen Leistungen als Folge des allgemeinen Spardrucks findet zwangsläufig auch im Fachgebiet der Altersmedizin statt. Die Diskussion wird dabei um das Thema der Altersdiskriminierung zusätzlich «bereichert». Es darf davon ausgegangen werden, dass der «agism», also die Diskriminierung wegen des Alters, und die Rationierung von medizinischen Leistungen miteinander in Verbindung stehen. Das Ende des Lebens ist dabei ein bequemes Argument für Kostenmanagement, indem vor allem technisch apparative Interventionen zur Unterlassung oder Durchführung als Rationierungsmassnahmen aufgeführt werden können. Die aktuelle Situation in der Schweiz lässt sich folgendermassen charakterisieren.

\section{These 1: Das Alter als Kriterium für allfällige Rationierungen von medizinischen Leistungen ist weder ein medizinisch begründbares noch ein ethisch vertretbares Kriterium}

Der Begriff der Rationierung stellt die ultima ratio der Zuteilung von Gütern in Kriegszeiten dar, und Zweifel und Telser fragen daher zu Recht [1], ob wir uns mit unseren betagten Mitbürgern im Kriegszustand befinden, dass wir sie von gewissen medizinischen Leistungen ausschliessen wollen. Das ethische Postulat der Gleichheit lässt es nicht $\mathrm{zu}$, älteren Menschen medizinische Leistungen vorzuenthalten. Das ökonomische Vorgehen, wo bei der Ressourcenallokation dort der Schwerpunkt gesetzt wird, wo das beste Kosten-Nutzen-Verhältnis erzielt werden kann, ist bei Betagten schwierig anwendbar. Wie lässt sich der Nutzen eines Lebensjahres beziffern, wenn die Person nicht mehr im Erwerbsleben steht? Repräsentative Umfragen in England für das Nationale Gesundheitssystem haben Mitte der 90er Jahre eine sehr niedrige Priorität für die Behandlung von Betagten ergeben. Auch in der Schweiz wurden ansatzweise - auf der Mikroebene - mit Zulassungsbeschränkungen mit Alterslimiten Erfahrungen gemacht (z. B. Verlegung auf Intensivstationen oder Dialyse, oder Zuweisung in eine Spezialklinik).

Anstelle des kalendarischen Alters werden häufig die Restlebenserwartung und die Qualität der zu erwartenden Lebensjahre, die sogenannten QUALYS (Quality Adjusted Life Years), zugezogen. Dabei steht im Zentrum - wiederum unter ökonomischer Sichtweise - die Frage, was kann oder darf die Verlängerung eines Lebens um ein Lebensjahr mit guter Lebensqualität kosten. Dabei wird der Nutzwert einer medizinischen Massnahme als Zugewinn an QUALYS, die durch die Durchführung oder Unterlassung dieser Massnahme realisiert werden, definiert. So wurden verschiedene Versuche unternommen, die Kosten pro qualitätsbereinigtes Lebensjahr zu beziffern. Auch das umstrittene Rationierungs- 
projekt in Oregon beinhaltete für die Formulierung von expliziten Rationierungskriterien Aspekte der Lebensqualität. Um diese ökonomische Sichtweise zu verfolgen, um insbesondere den finanziellen Wert eines Lebensjahres zu beziffern, wurden Vergleiche mit anderem Konsumverhalten durchgeführt (Risiko für Wohnungsbrände oder Verkehrsunfälle, verbunden mit der Zahlungsbereitschaft für Rauchdetektoren oder Zustimmung zu Geschwindigkeitsbeschränkungen auf Autobahnen).

Bis jetzt konnte kein Gesundheitssystem überzeugend darlegen, anhand welcher Kriterien eine gerechte Erfassung der Lebensqualität erfolgen könnte. Insbesondere sind bei solchen Diskussionen die direkt betroffenen, also in unserem Fall die 80jährigen, nicht involviert worden. Interessant sind denn auch die Stellungnahmen von pensionierten Personen aus Zürich, die im Anschluss an eine Vortragsreihe «Rationierung im Gesundheitswesen: Betagte als Opfer» differenziert über komplexe Mittelallokationsentscheide urteilten. Diese Personen haben auch Höchstbetagten die Kompetenz zur Entscheidungsfindung zugesprochen [2].

Untersuchungen zeigen, dass die letzten zwei Lebensjahre eines Patienten, der mit 65 Jahren stirbt, wesentlich mehr Krankheitskosten auslösen als für die letzten zwei Lebensjahre eines Patienten, der mit 95 Jahren stirbt, aufgewendet werden [3]. Dies kann ein Hinweis dafür sein, dass informelle, implizite Kriterien, vielleicht ein langjähriges Arzt-/Patienten-/Angehörigenverhältnis zugrunde liegen und so die Entscheidungsfindung in gegenseitiger Übereinkunft gefunden werden kann. Prof. Stella Reiter-Theil, Forscherin des Instituts für angewandte Ethik und Medizinethik, formuliert es so: «Für sich genommen, darf das Alter kein Kriterium für das Vorenthalten von medizinischen Angeboten sein, denn das wäre genau so ungerecht wie etwa die gesellschaftliche Position als Kriterium.» [4]

\section{These 2: Als Folge von allgemeinem Spardruck hat sich die implizite Rationierung breitgemacht. Die Altersmedizin ist davon betroffen}

Markenzeichen der expliziten Rationierung sind: Die Kriterien sind klar definiert, die diesbezügliche Entscheidungsfindung ist transparent und die Verantwortlichen der Kriterienerarbeitung sind ersichtlich. Entsprechend sind bei der impliziten Rationierung keine Verantwortlichen sichtbar, die Kriterien und die diesbezügliche Entscheidungsfindung verbleiben im dunkeln.

Reduzierte Bettenzahlen, Abbau von Pflegepersonal im stationären Bereich und verkürzte Hospitalisationsdauer sind die Folgen des Spardruckes im stationären Bereich. Die Verlagerung der Ressourcen, weg von den Personalkosten hin
2 Schelling HR, Wettstein A. Einstellung von Seniorinnen und Senioren zur Rationierung im Gesundheitswesen - vor und nach einer Vorlesungsreihe. Praxis 2000;89:1200-10.

3 Lubitz J, Beebe J, Baker C. Longevity and Medicare expenditures. N Engl J Med 1995;332(15):999-1003.

4 Dieffenbacher C. «Patientenwünsche wichtiger geworden». Interview mit Prof. Stella ReiterTheil. Uni Nova Wissenschaftsmagazin der Universität Basel 90, März 2002.

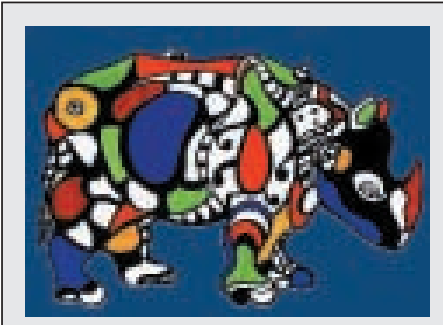

\section{Jahresversammlung der Schweizerischen Gesellschaft für Innere Medizin sowie Jahrestagung der Schweizerischen Gesellschaft für Hämatologie Schweizerischen Fachgesellschaft für Geriatrie Sektion Klinische Pharmakologie und Toxikologie Schweizerischen Hypertonie Gesellschaft}

Ort und Datum: Basel, Mittwoch, 21. Mai, bis Freitag, 23. Mai 2003

Der Klinische Track soll Gelegenheit geben, nicht nur das Neueste, sondern das Entscheidendste zu erfahren.

Clinical year in review: Exponenten aus verschiedenen Fachgebieten der Medizin bieten einen Überblick über neueste wissenschaftliche und klinisch relevante Erkenntnisse ihres Faches.

Clinical pearls: Chefärzte der Nordwestschweiz präsentieren ausgewählte Fälle.

Workshops: Grundversorger und Experten bearbeiten gemeinsam mit dem Publikum ein Thema in kleinen Gruppen.

Skill lab: Informatik, Blutdruckmessung, Gastroskopie, Bronchoskopie, Inhalationstechniken, Prostatapalpation, Selbststudiumprogramme in Radiologie, Ergonomie am Arbeitsplatz.

Sachverstandprüfung im Strahlenschutz BAG: 21. Mai, 11.45 Uhr.

Administratives Sekretariat: Jahresversammlung SGIM 2003, c/o AKM Congress Service, Postfach, 4005 Basel, Tel. 06168677 11, Fax 06168677 88, E-Mail: info@akm.ch

Website mit weiteren Infos: www.akm.ch/sgim 2003 
5 Schopper D, Baumann-Hölzle R, Lange F, Beeler I, Roth F, Tanner M. Mittelverteilung im schweizerischen Gesundheitswesen am Beispiel der Kantone Zürich und Basel-Stadt. Schweiz Ärztezeitung 2002;83(44):2364-71.

6 Sommer JH. Muddling through elegantly: Rationierung im Gesundheitswesen. Basel: EMH Schweizerischer Ärzteverlag; 2001. zu medizinischer High-Technologie ist gerade für die Altersmedizin deletär. Die in der Geriatrie gebräuchlichen Instrumente, wie beispielsweise das geriatrische Assessment, benötigen Personalressourcen und nicht High-Technologie. Eine Untersuchung in Basel und Zürich hat gezeigt, dass die Ressource «Menschlichkeit» durch die als Folge des Spardrucks erfolgte (implizite) Rationierung in Gefahr ist [5].

Die gleiche Untersuchung zeigt auch, dass durch den allgemeinen Trend, stationäre Kapazitäten abzubauen, ein Ausweichen in die Ambulanz stattfindet. Für betagte Patienten bedeutet dies häufig: übereilter Spitalaustritt, rascher Ortswechsel, schwieriges Zurechtfinden in neuen Situationen und entsprechend rasch erneute Rehospitalisation (sogenannter enterogeriatrischer Kreislauf oder Drehtüreffekt).

Es ist davon auszugehen, dass implizite Rationierungsmassnahmen gerade im Bereich der Altersmedizin keine Kosten sparen werden.

Die in der Geriatrie tätigen Berufsgruppen sind sich dieser Problematik sehr wohl bewusst.
Dank Untersuchungen und entsprechender Forschung wissen wir, welche Interventionen und Massnahmen auch bei Hochbetagten sinnvoll und erfolgsversprechend sind und daher niemandem a priori vorenthalten werden können. Natürlich braucht es dazu das nötige geriatrische Know-how, um im Kontext des allgemeinen Spardruckes auf die medizinischen Bedürfnisse auch der (Randgruppe der) Hochbetagten hinzuweisen. Vielleicht muss unsere Strategie weiterhin etwa so lauten, wie sie H. Sommer zitiert: «Muddling through Elegantly» oder «elegantes Durchwursteln» [6].

Am diesjährigen Jahreskongress der Schweizerischen Gesellschaft für Innere Medizin, der wie gewohnt zusammen mit der Schweizerischen Fachgesellschaft für Geriatrie in Basel stattfindet, wird mein Kollege Dr. Charles Chappuis auf Aspekte der Rationierung in der Geriatrie eingehen. Hier soll schon der Appetit geweckt werden, sich mit diesem Thema auseinanderzusetzen und natürlich die erwähnte Veranstaltung zu besuchen. 